\title{
Element size effect on the analysis of heavy-duty machine cross-rail Jianhua Wang ${ }^{1}$,Jianke Chen ${ }^{1, a}$,Tieneng Guo ${ }^{1}$, Bin Song ${ }^{2}$, Dongliang Guo ${ }^{3}$ \\ ${ }^{1}$ Beijing University of Technology , Beijing 100124 , China; \\ ${ }^{2}$ Institute of Electrical Engineering Chinese Academy of Sciences, Beijing 100080 , China; \\ ${ }^{3}$ Capital Spaceflight Machinery Company , Beijing 100076, China \\ a chenjianke@emails.bjut.edu.cn
}

Keywords: Heavy-duty machine cross-rail , Finite element , Element size.

\begin{abstract}
Heavy-duty gantry machine plays an important role in the energy, shipbuilding, aerospace, transportation and military industry and other pillar industries, and is a manifestation of national equipment manufacturing capacity. Cross-rail is one of the key structural components, and its natural frequency affects the dynamic performance of the machine. It is difficult to obtain the accurate analysis of natural frequency by using the analytical method because of the complex internal structure. The finite element method is a general method to obtain natural frequency, but, is also difficult to obtain the precise result, because the analysis result depends on many factors, such as meshing manners, the constraint conditions, joint parameters, damping, and so on. The density of mesh is an important factor to affect the analysis result especially. Theoretically, the smaller element, the closer to the true result. But more dense grid could sharply increase the degree of freedom, and result in the insufferable solving speed in the analysis of the cross-rail. So to find a balance point between the speed and the accuracy becomes very meaningful. In this paper, the modal analysis was carried out on the cross-rail. The paper focus on exploring the influence of different element size on the results of the analysis. In order to verify the correctness of the modeling, the modal testing is carried on the cross-rail, and the experimental result is adopted to prove the correctness of finite element modeling.
\end{abstract}

\section{Cross-rail free modal finite element analysis}

\subsection{The cross-rail profile:}

Cross-rail on this study is about 15 meters, and the quality is 99.716 tons, as shown in figure 1-1.The global coordinate system is as following. Anti-gravity is the $\mathrm{Z}$ direction, $\mathrm{Y}$ is the longitudinal direction, front and rear direction is $\mathrm{X}$ direction.

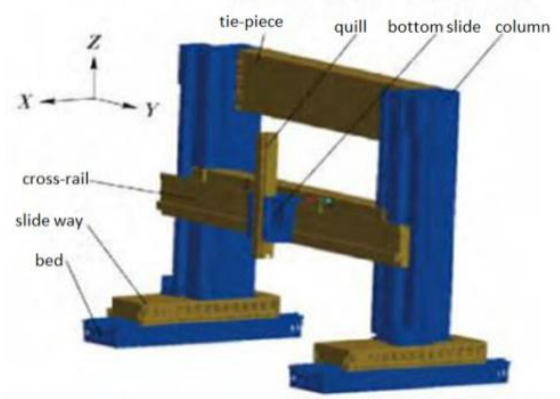

Fig. 1-1 CNC heavy-duty gantry machine and global coordinates

The cross-rail material is QT600. The property of the cross-rail material is as showing in table 1-1

Table 1-1 Cross-rail material parameters

\begin{tabular}{ccc}
\hline Density $\left(\mathrm{kg} / \mathrm{m}^{3}\right)$ & Young's modulus (GP) & Poisson's ratio \\
\hline 7200 & 174 & 0.275 \\
\hline
\end{tabular}




\subsection{FEM element selection:}

The analysis tool of FEM is Ansys. Solid185 element is adopted to mesh the cross-rail. Solid185 entity structure applicable to the general three-dimensional structure modeling, it can be to prism, tetrahedron and pyramid for degradation, the element is shown in figure 1-2.

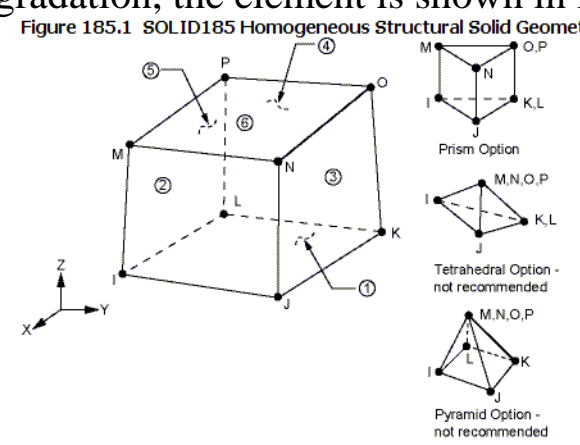

Fig.1-2 Solid185 element and degradation

Solid185 element is defined by eight nodes, and each node has the X, Y, Z three directions of the translational degrees of freedom. The element has a super elasticity, stress players, creep, large deformation and large strain capabilities. Stress output of Solid185 elements as shown in figure 1-3.

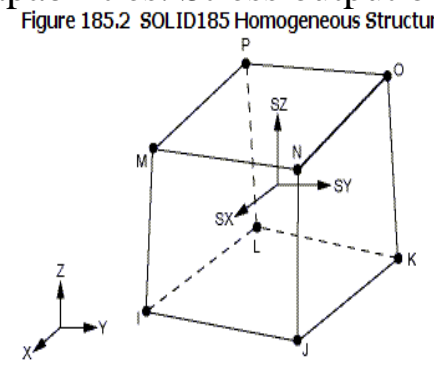

Stress directions shown are for global directions.

Fig. 1-3 Stress output direction of the Solid185 element (SX, SY, SZ)

\subsection{Effect of element size on modal analysis:}

Adopting the automatic meshing method. In the condition of completely free-free, modal analysis was carried out on the cross-rail, not imposed any constraint. Since cross-rail is in a free-free state, the first six orders vibration belongs to the rigid body motion, so the natural frequency is zero. Beginning with 7th frequency values, namely that the 7th order as the first order modal. Studies have shown that the size of the element will largely influence the finite element simulation result. It is necessary to control the grid size to achieve ideal result. At first, taking the element size of $30 \mathrm{~mm}$, and the first 5 order modal simulation result is shown in figure1-4.

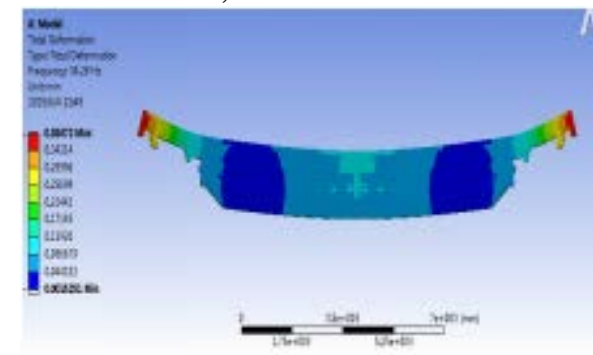

a) First-order mode shape

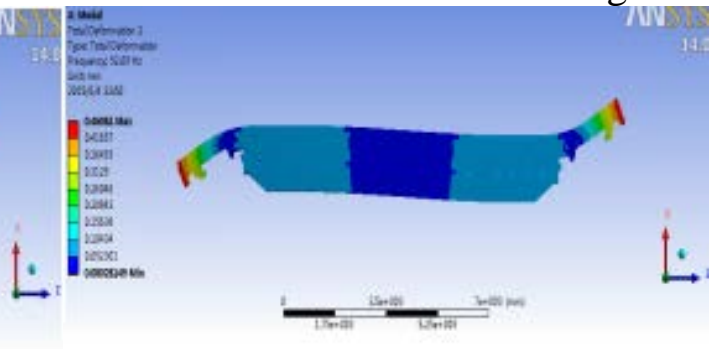

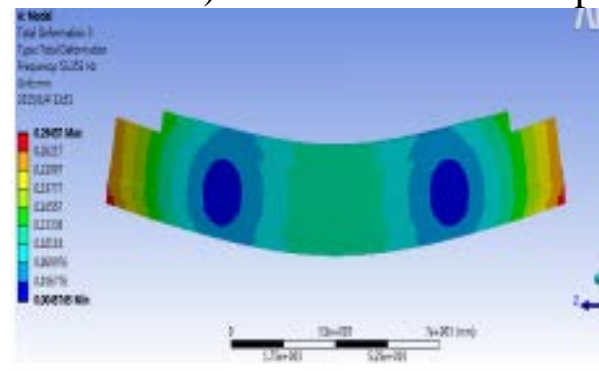

c) Third-order mode shape b) Second-order mode shape

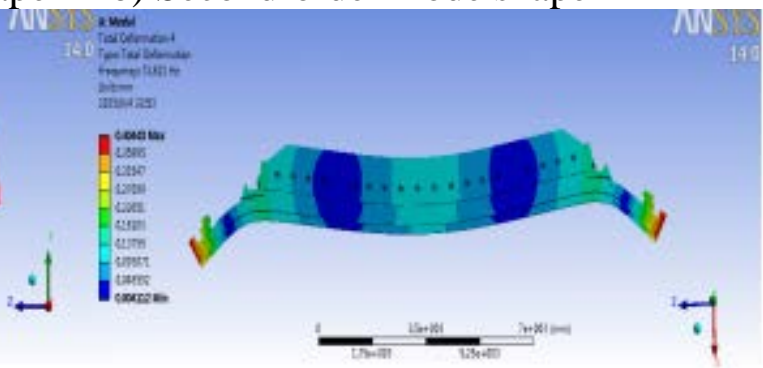

d) Fourth-order mode shape 


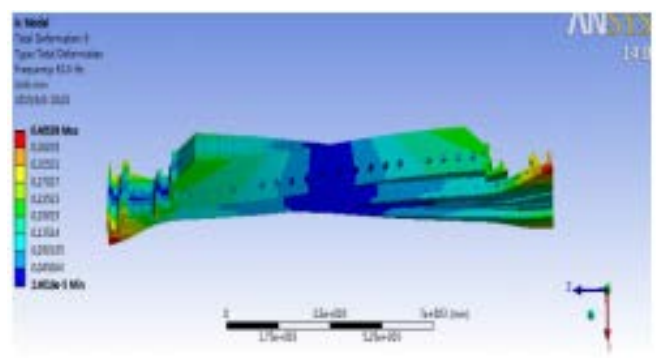

e) Fifth-order mode shape

Fig. 1-4 First 5 order mode simulation results of cross-rail

Then element size selection in turn: $200 \mathrm{~mm}, 150 \mathrm{~mm}, 100 \mathrm{~mm}, 90 \mathrm{~mm}, 80 \mathrm{~mm}, 70 \mathrm{~mm}, 60 \mathrm{~mm}, 50$ $\mathrm{mm}, 45 \mathrm{~mm}, 40 \mathrm{~mm}, 35 \mathrm{~mm}, 30 \mathrm{~mm} .12$ groups of data is obtained by 12 kinds of element size. The element size on the result of modal analysis is shown in figure 1-5.

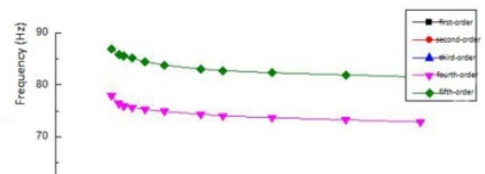

Fig.1-5 Cross-rail frequency change with element number

Simulation data shown in table 1-2.

Table 1-2 Modal frequency under different meshing method

\begin{tabular}{|c|c|c|c|c|c|c|c|}
\hline \multirow{2}{*}{$\begin{array}{c}\text { Element size } \\
(\mathrm{mm})\end{array}$} & \multirow{2}{*}{$\begin{array}{c}\text { Node } \\
\text { number }\end{array}$} & \multirow{2}{*}{$\begin{array}{c}\text { Element } \\
\text { number }\end{array}$} & \multicolumn{5}{|c|}{ Each order modal frequency $(\mathrm{Hz})$} \\
\hline \cline { 5 - 8 } & 300657 & 167383 & 35.955 & 54.911 & 57.033 & 78.808 & 87.228 \\
\hline 150 & 325315 & 183397 & 35.781 & 54.989 & 56.256 & 77.895 & 86.860 \\
\hline 100 & 359314 & 201989 & 35.483 & 54.707 & 54.994 & 76.367 & 85.826 \\
\hline 90 & 387562 & 218533 & 35.396 & 54.625 & 54.713 & 75.906 & 85.509 \\
\hline 80 & 422726 & 238682 & 35.290 & 54.402 & 54.512 & 75.649 & 85.107 \\
\hline 70 & 493003 & 277825 & 35.142 & 54.121 & 54.302 & 75.264 & 84.441 \\
\hline 60 & 582564 & 327561 & 34.984 & 53.851 & 54.072 & 74.911 & 83.777 \\
\hline 50 & 739184 & 416595 & 34.767 & 53.359 & 53.829 & 74.327 & 83.035 \\
\hline 45 & 878327 & 498193 & 34.656 & 53.066 & 53.727 & 73.997 & 82.717 \\
\hline 40 & 1044618 & 595583 & 34.558 & 52.804 & 53.605 & 73.679 & 82.326 \\
\hline 35 & 1339415 & 768650 & 34.426 & 52.480 & 53.466 & 73.277 & 81.891 \\
\hline 30 & 1652491 & 950384 & 34.280 & 52.090 & 53.352 & 72.821 & 81.500 \\
\hline
\end{tabular}

\section{The experiment measuring cross-rail modal data}

Test location: Some machine tool plant

Test content: The natural frequency, vibration mode and damping ratio of the cross-rail

Modal testing equipment: The experiment use the exciter method to motivate, vibration exciter can make uniform distribution of energy in the structure. Incentives for a point of the cross-rail, you can get multiple batches of response data, so you can get a very good response data in a very short period of time. Adopting sinusoidal scanning incentive structure, HEV - 200 vibration exciter - the largest exciting force is $200 \mathrm{~N}$. Using LMS vibration test and analysis system and its supporting PCB acceleration sensor. The principle of testing is shown in figure 2-1. 

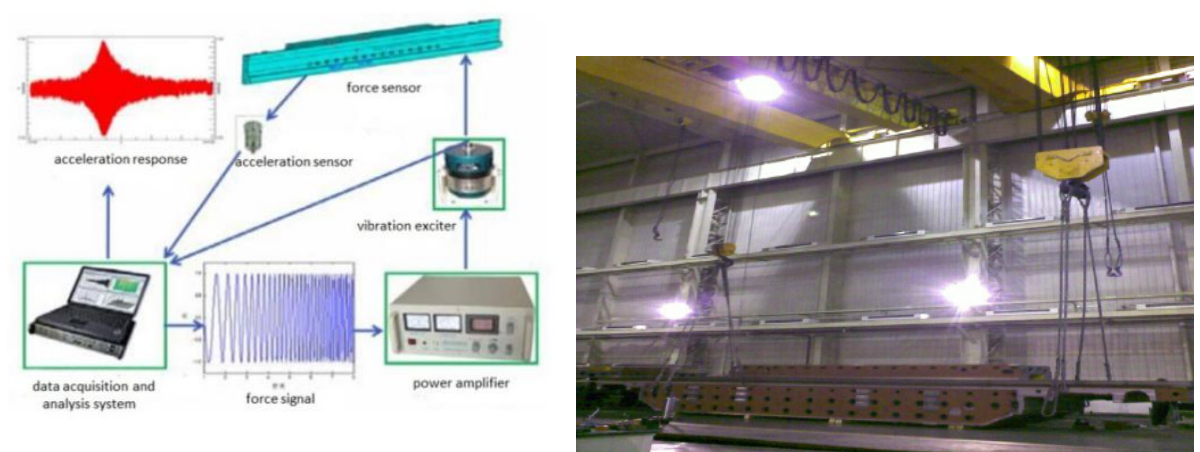

Fig. 2-1 Sine scanning excitation test principle

Cross-rail is lifted by the crane so as to make it in the free state . Incentive cross-rail with the front and rear position. The placement of the cross-railvexciting point and measuring points as shown in figure 2-2.

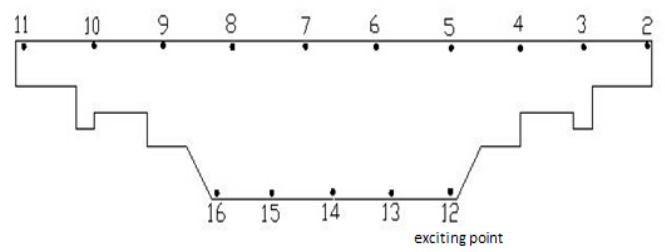

Fig. 2-2 The placement of the cross-rail v exciting point and measuring points

Using the dynamic frequency sweep method for scanning cross-rail. First is sweep roughly, Set incentive frequency range as follows: $5 \mathrm{~Hz}-100 \mathrm{~Hz}$, exciting force is about $120 \mathrm{~N}$, scanning speed is $0.08 \mathrm{~Hz} / \mathrm{s}$. Probably sweep time required to complete a measurement for 20 minutes. Through software for frequency response function of LSCE computing the natural frequencies of the cross-rail, further to the damping of the cross-rail at the natural frequency. Combining all the stimulation test results, the natural frequency of the cross-rail and damping ratio are shown in table 2-1:

Table 2-1 Natural frequency and damping ratio

\begin{tabular}{cc}
\hline Natural frequency $(\mathrm{Hz})$ & Damping ratio \\
\hline 31.72 & 0.0021 \\
48.70 & 0.0053 \\
54.76 & 0.0020 \\
74.84 & 0.0013 \\
82.12 & 0.0025 \\
\hline
\end{tabular}

\section{The experimental data and the simulation results}

\subsection{Contrast mode}

The result of the experiment and the simulation result are shown in figure 3-1.The left is the simulation result and the experiment result is on the right. 


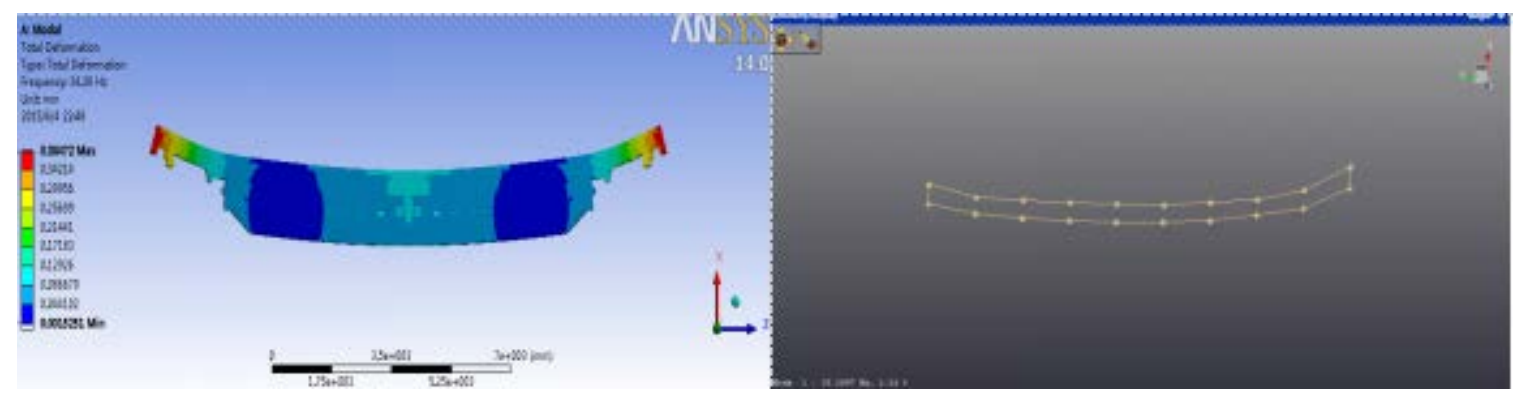

a) First order mode shape

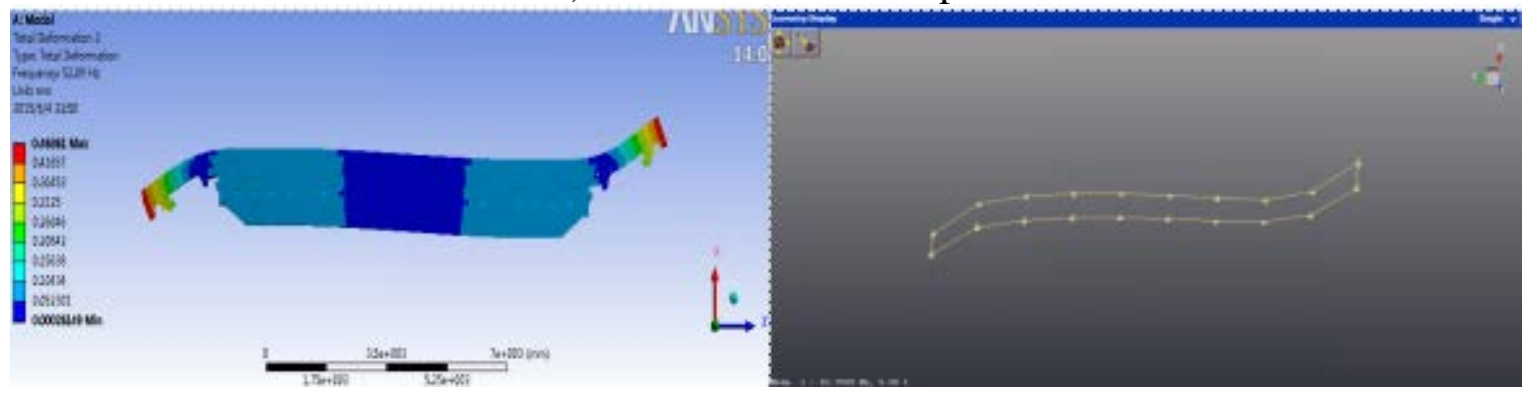

b) Second order mode shape

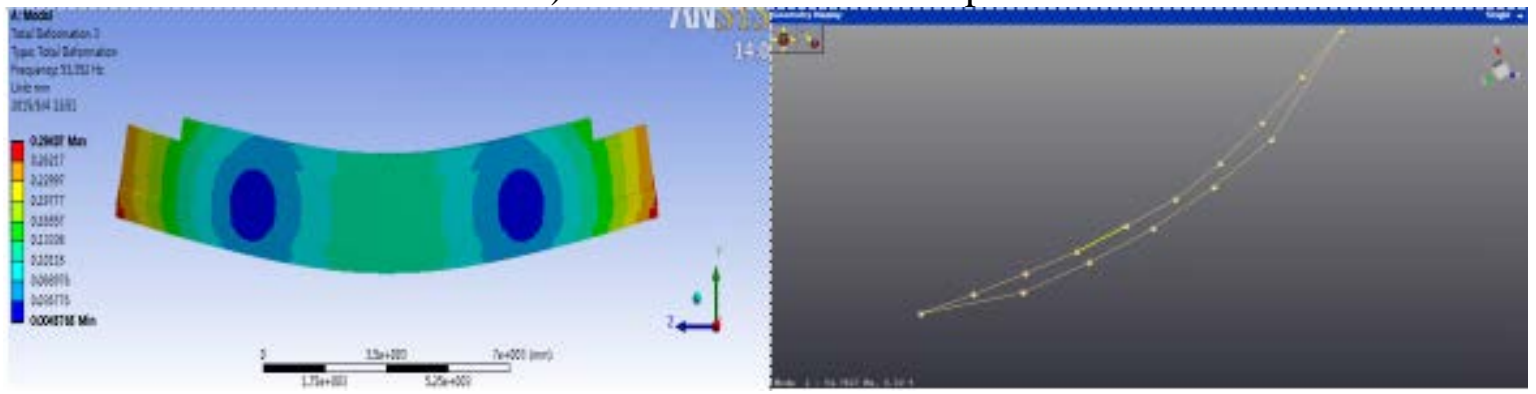

c) Third order mode shape

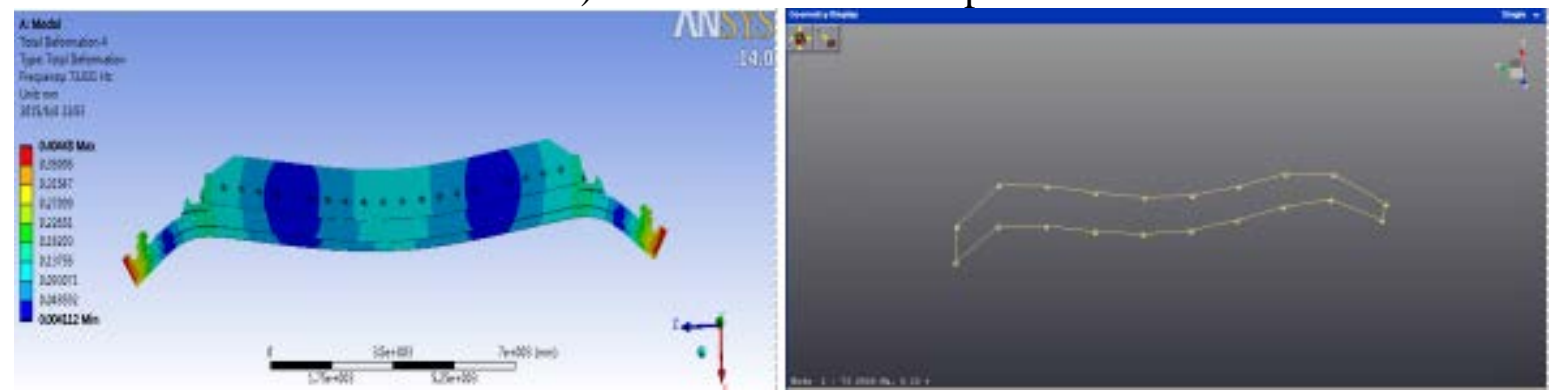

d) Fourth order mode shape

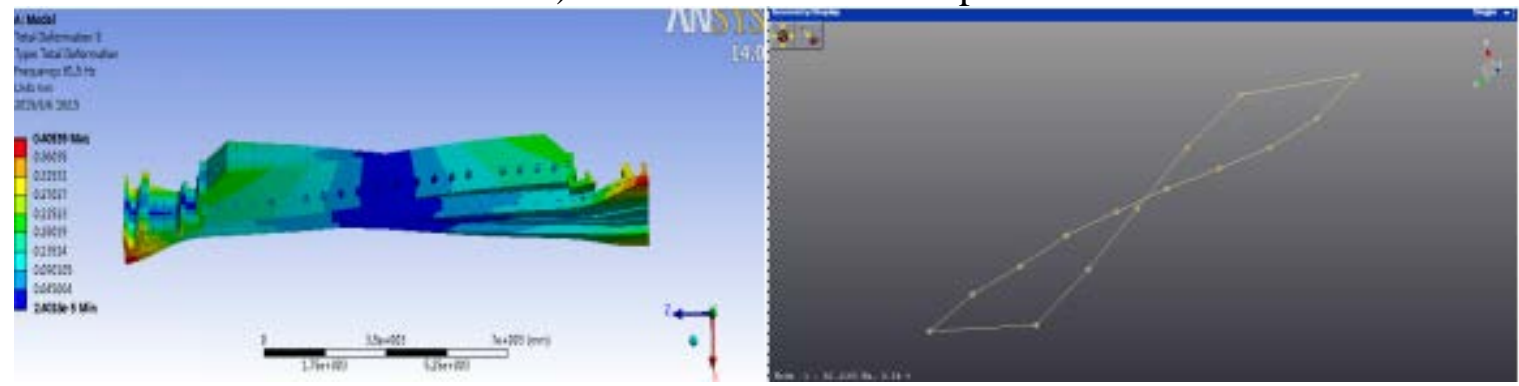

e) Fifth order mode shape

Fig. 3-1 Cross-rail first 5 order modal experiment and the simulation results

3.2 Contrast the simulation natural frequency and the natural frequency of the experiment

It is easy to see that two kinds of modes are basically the same. Then contrasting the natural frequency error obtained by simulation and experimental data, the results are shown in Table 3-1 and Table 3-2 
Table 3-1 Element size is $30 \mathrm{~mm}$ simulation error

\begin{tabular}{ccc}
\hline The experimental value $(\mathrm{Hz})$ & The simulation value $(\mathrm{Hz})$ & The percentage error $(\%)$ \\
\hline 31.72 & 34.28 & 8.07 \\
48.70 & 52.09 & 6.96 \\
54.76 & 53.35 & -2.57 \\
74.84 & 72.82 & -2.70 \\
82.12 & 81.50 & -0.7 \\
\hline
\end{tabular}

Table 3-2 Element size as the default simulation error

\begin{tabular}{ccc}
\hline The experimental value $(\mathrm{Hz})$ & The simulation value $(\mathrm{Hz})$ & The percentage error $(\%)$ \\
\hline 31.72 & 36.92 & 16.39 \\
48.70 & 55.45 & 13.86 \\
54.76 & 60.89 & 11.19 \\
74.84 & 83.51 & 11.58 \\
82.12 & 89.61 & 9.12 \\
\hline
\end{tabular}

It can be seen that the result between the experimental and simulation results match better, when the element size is equal to $30 \mathrm{~mm}$.

\section{Summary}

The element size effect of modal analysis on the cross-rail is described. First, different element size is adopted to mesh the model of cross-rail, and then the cross-rail mode is analyzed. The analysis result is compared with the experimental results. When the element size decreases, namely the increase of grid density, each order natural frequency of the cross-rail will decrease, and more and more close to the experimental value. When the size is close enough, the simulation results will converge to the true value. When the element size is at the default value in Ansys, the first-order natural frequency and the error between the experimental value is as high as $16.39 \%$. And when the element size is $30 \mathrm{~mm}$, the natural frequency error fell by nearly half. Thus the element size had a great influence on the result of cross-rail modal analysis. At the same time, the simulation and experimental results in terms of natural frequencies or modes are basically the same, so the cross-rail finite element modeling method is feasible. The smaller the element size need the higher requirement for equipment processor, The element size of $30 \mathrm{~mm}$ has been able to better match to the experimental results, and the subsequent analysis adopt the size of $30 \mathrm{~mm}$.

\section{Acknowledgments}

This work was financially supported by "the National Science and Technology Major Project of the Ministry of Science and Technology of China (2014ZX04001-171)”

\section{Reference}

[1] CAO Ming, SONG Chun-ming, ZHANG Dong-sheng, TONG Guo-rong. Based on the ANSYS determinate cross-rail gantry machine horizontal nicole mechanical characteristics analysis and structural optimization [J]. Mechanical engineers, 2014, 10:p112-114

[2]GUO Tie-neng, LI Ling, CAI Li-gang, LIU Zhi-feng, ZHAO Yong-sheng, YANG Kun. Identifying mechanical joint dynamic parameters based on measured frequency response functions [J]. Vibration and shock,2011,05:p69-72

[3] ZHANG Guo-rui. Finite element method (fem) [M]. Beijing: mechanical industry publishing house, 1999

[4]LI Yang, YANG Qing-dong,LIU Guo-qing, Parameter identification and dynamics of gantry NC machine tool beam part $[\mathrm{J}]$. Journal of Beijing university of information science and technology 
(natural science edition), 2010,03, Vol.25 No.1

[5]GUO Tie-neng, YU Xue-li, LI Fu-ping, CAI Li-gang, GUO Dong-liang Modeling Study of the CNC Heavy-duty Gantry Machine Coupling With the Characteristics of the Joint Surfaces [J]. Journal of Beijing university of technology,2013,03,Vol.39 No.3

[6]LIU Zhi-feng, SONG Bin, GUO Tie-neng, CAI Li-gang, GUO Dong-liang, WANG Feng Influences of the Bolted Joints on Dynamic Characteristics of Multi-section Cross-rail [J]. Journal of Beijing university of technology ,2012,07,Vol.38 No.7 